\title{
The Floer Center of Geometry
}

Alberto Abbondandolo (Ruhr University, Bochum, Germany)

The Floer Center of Geometry has been active at the Ruhr University of Bochum since 2011. It is named after Andreas Floer, who was first a student and later a professor at the Faculty of Mathematics in Bochum and whose ideas profoundly influenced the fields of symplectic geometry and low dimensional topology.

\section{Andreas Floer}

Andreas Floer was born in 1956 in the German town of Duisburg, where the river Ruhr meets the Rhine, in the middle of a densely populated mining and industrial area. At the time of his birth, there was not a single university in the whole Ruhr area but that situation was soon going to change. Local and national governments had understood that coal and heavy industry were not forever and had started to diversify. One of the first effects of this diversification was the foundation of the Ruhr University of Bochum in 1962, the "oldest of the new universities", that is, the first of the new public universities that were built in Germany after World War II. Classes in Bochum started in 1965 and, a decade later, Floer enrolled as a student in mathematics. He specialised in algebraic topology and dynamical systems, two fields that were investigated in Bochum under the chairs of Heiner Zieschang and Eduard Zehnder. He received his diploma in 1982 under the supervision of Ralph Stöcker.

He then spent a year and a half in Berkeley, where he started his PhD studies working with Clifford Taubes and Alan Weinstein. He then returned to Bochum, where he received his doctoral degree in 1984 under the supervision of Eduard Zehnder. He later had research positions at SUNY in Stony Brook and at the Courant Institute of New York University, before becoming an assistant professor at Berkeley in 1988. In the Autumn of 1990, he returned to Bochum as a full professor. He tragically took his life on 15 May 1991.

Floer's work had a deep impact in symplectic geometry, gauge theory and low dimensional topology. Most of the recent progress in these fields would be simply unthinkable without the seminal ideas that shape what is now known as Floer homology. Floer homology is not a single theory but rather a new approach to the study of critical points of certain geometric functionals and a way of producing algebraic invariants out of them.

The first version of Floer's theory was motivated by the Arnold conjecture on the number of fixed points of Hamiltonian diffeomorphisms. In order to attack this conjecture, one has to prove lower bounds on the number of critical points of a functional (the action functional from Hamiltonian mechanics), which presents severe analytical difficulties. It is a functional defined on the space of closed loops on a symplectic manifold and its second differential at critical points is strongly indefinite, meaning that maximal subspaces on which this bilinear form is positive or negative definite are both infinite dimensional. Floer observed that a suitable gradient equation for this functional is given by an elliptic partial differential equation, which is a lower order perturbation of the nonlinear Cauchy-Riemann equation that Gromov had introduced and studied in 1985. Starting from this observation, Floer developed a Morse theory for the action functional based on an algebraic counting of the spaces of finite energy solutions of this elliptic equation, and eventually proved the Arnold conjecture for a class of closed symplectic manifolds. Together with Helmut Hofer, he also realised that his Floer homology could also be used to produce new powerful invariants for domains inside symplectic manifolds.

Floer soon realised that his ideas could also be applied to the study of the Chern-Simons functional, a functional on the space of $\mathrm{SU}(2)$ and $\mathrm{SO}(3)$-connections over a three-manifold whose critical points are flat connections. This led him to the definition of the instanton Floer homology of a three-manifold, a theory which plays a major role in the study of four-manifolds with boundary.

A quarter of a century after Floer's work, his ideas permeate symplectic geometry and low dimensional topology. Lagrangian intersection Floer homology, contact homology, embedded contact homology, Heegaard Floer homology and Seiberg-Witten Floer homology are just a few of the theories that grew out of the work of this extremely talented mathematician.

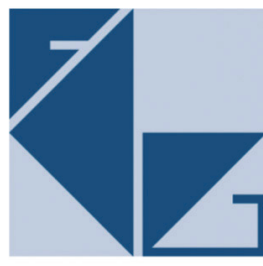

\section{FLOER} CENTER OF GEOMETRY

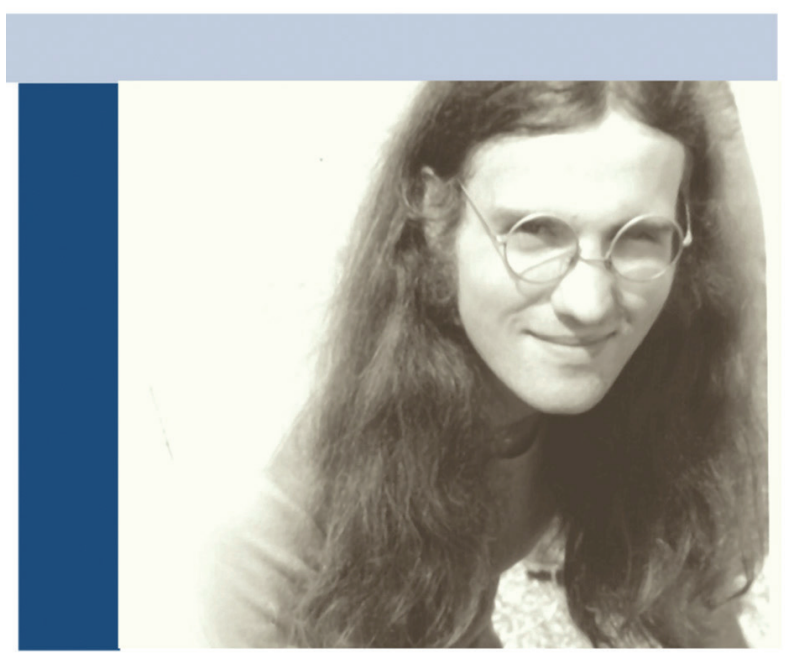




\section{The Floer Center}

The Floer Center of Geometry was opened in Bochum in 2011, 20 years after Andreas Floer's tragic death. It is based at the Faculty of Mathematics of the University of Bochum and brings together the research activity of professors, postdocs and $\mathrm{PhD}$ students working in algebra, algebraic geometry, complex geometry, differential geometry, dynamical systems, symplectic geometry and topology.

The opening ceremony took place in December 2011, with colloquium talks by Helmut Hofer, Stefan Nemirovski and Andrew Ranicki, who were introduced by Gerd Laures (initiator of the foundation of the Floer Center of Geometry and its first director until 2013).

One of the main events of the Floer Center of Geometry is the annual Floer Lectures, which, over the last few years, have been given by Kai Cieliebak, Alex Ritter, Claude Viterbo, Frol Zapolski, Yakov Eliashberg, Hansjörg Geiges, Stefan Nemirovski, Ralph Cohen, Thomas Kragh, Michael Weiss and Thomas Willwacher.

In February 2017, the Floer Center of Geometry hosted a very special edition of "Geometric Dynamic Days", an annual event involving several German universities. The speakers of this edition, Helmut Hofer and Eduard Zehnder, have been close collaborators and friends of Andreas Floer since his undergraduate years. Their lectures on "Pseudoholomorphic Curves in Hamiltonian Dynamics and Symplectic Geometry" and "The beginnings of symplectic topology at the RUB" can be watched online at

http://www.ruhr-uni-bochum.de/ffm/Lehrstuehle/

Lehrstuhl-VII/gdd17.html.

The next edition of the Floer Lectures will take place on 21-22 June 2018, with four talks by Victor Guillemin and Paul Seidel.
Since 2017, the Floer Center of Geometry offers Floer Postdoctoral Research Fellowships: three-year postdoc positions for young mathematicians working in the core fields of the centre. The first postdoctoral fellow Lara Simone Suarez, an expert in Lagrangian Floer homology and Lagrangian cobordism, was hired in October 2017.

The Floer Center of Geometry is funded by the University of Bochum through the Faculty of Mathematics and by diverse grants of the Deutsche Forschungsgemeinschaft (DFG). It serves also as a platform to coordinate the activities of the Collaborative Research Centre on "Symplectic Structures in Geometry, Algebra and Dynamics", a collaboration scheme funded by the DFG and based at the Universities of Bochum and Cologne.

\section{References}

[1] Floer Center of Geometry's web site. http://www.floer.rub.de/index.html.en.

[2] S. K. Donaldson. On the work of Andreas Floer. Jahresber. Deutsch. Math.-Verein., 95(3):103-120, 1993.

[3] Helmut Hofer, Cliord H. Taubes, Alan Weinstein, and Eduard Zehnder, editors. The Floer Memorial Volume, Progress in Mathematics Vol. 133. Birkhäuser Verlag, Basel, 1995.

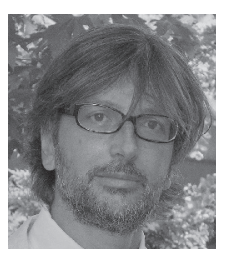

Alberto Abbondandolo [alberto.abbondandolo@rub.de] is a professor of analysis at the Ruhr University of Bochum and the Director of the Floer Center of Geometry. He works in symplectic geometry, dynamical systems and global analysis. 